\title{
Partial Regularity of solutions to the Four-Dimensional Navier-Stokes Equations
}

\author{
Hongjie Dong and Xumin Gu
}

Communicated by Y. Charles Li, received December 11, 2013.

\begin{abstract}
In this paper, we consider suitable weak solutions of incompressible Navier-Stokes equations in four spatial dimensions. We obtain two $\varepsilon-$ regularity criteria in terms of certain scale-invariant quantities. As a consequence, we show that the two-dimensional space-time Hausdorff measure of the set of singular points is equal to zero.
\end{abstract}

\section{Contents}

1. Introduction

2. Notation and Setting $\quad 56$

3. The proof 57

$\begin{array}{ll}\text { Acknowledgements. } & 68\end{array}$

$\begin{array}{ll}\text { References } & 68\end{array}$

\section{Introduction}

In this paper we consider the incompressible Navier-Stokes equations in four spatial dimensions with unit viscosity and an external force:

$$
\begin{aligned}
u_{t}+u \cdot \nabla u-\Delta u+\nabla p & =f, \\
\nabla \cdot u & =0
\end{aligned}
$$

in a bounded cylindrical domain $Q_{T} \equiv \Omega \times(0, T)$, where $\Omega \subset \mathbb{R}^{4}$. We are interested in the partial regularity of suitable weak solutions $(u, p)$ to $(1.1)-(1.2)$.

We say that a pair of functions $(u, p)$ is a suitable weak solution to (1.1)-(1.2) in $Q_{T}$ if $u \in L_{\infty}\left(0, T ; L_{2}\left(\Omega ; \mathbb{R}^{4}\right)\right) \cap L_{2}\left(0, T ; W_{2}^{1}\left(\Omega ; \mathbb{R}^{4}\right)\right)$ and $p \in L_{3 / 2}\left(Q_{T}\right)$ satisfy

1991 Mathematics Subject Classification. 35Q30, 76D03, 76D05.

Key words and phrases. Navier-Stokes equations, partial regularity, Hausdorff's dimension.

(C)2014 International Press 
(1.1)-(1.2) in the weak sense and additionally they satisfy the generalized local energy inequality:

$$
\begin{aligned}
\underset{0<s \leq t}{\operatorname{ess} \sup _{\Omega}} \int_{\Omega}|u(x, s)|^{2} \psi(x, s) d x+2 \int_{Q_{t}}|\nabla u|^{2} \psi d x d s \\
\leq \int_{Q_{t}}|u|^{2}\left(\psi_{t}+\Delta \psi\right)+\left(|u|^{2}+2 p\right) u \cdot \nabla \psi+2 f \cdot u \psi d x d s
\end{aligned}
$$

for any non-negative functions $\psi \in C_{0}^{\infty}\left(Q_{T}\right)$. We prove that for any suitable weak solution $(u, p)$, the two dimensional space-time Hausdorff measure of the set of singular points is equal to zero.

The problem of the global regularity of solutions to the Navier-Stokes equations in three and higher space dimensions is a fundamental question in fluid dynamics and is still widely open. Meanwhile, many authors have studied the partial regularity of solutions. In three dimensional case, Scheffer established various regularity results for weak solutions in $[\mathbf{1 3}, \mathbf{1 4}]$. In a celebrated paper $[\mathbf{1}]$, Caffarelli, Kohn, and Nirenberg introduced the notion of suitable weak solutions, which satisfy a local energy inequality. They proved that for any suitable weak solution, there is an open subset where the velocity field $u$ is regular and the 1D Hausdorff measure of the complement of this subset is equal to zero. In [12], Lin gave a more direct and simplified proof of Caffarelli, Kohn and Nirenberg's result with zero external force, under a slightly different assumption on the pressure term. Ladyzhenskaya and Seregin provided yet another and detailed proof of Caffarelli-Kohn-Nirenberg's and Lin's results in [10]. We also refer the reader to Tian and Xin [18], Seregin [16], Gustafson, Kang, and Tsai [5], Vasseur [19], Kukavica [9], and the references therein for extended results.

For the four or higher dimensional Navier-Stokes equations, the problem is more super-critical. In [15], Scheffer showed that there exists a weak solution $u$ in $\mathbb{R}^{4} \times \mathbb{R}^{+}$, which may not satisfy the local energy inequality, such that $u$ is continuous except for a set whose 3D Hausdorff measure is finite. In [2], the first author and D. Du showed that for any local-in-time smooth solution to 4D Navier-Stokes equations, the 2D Hausdorff measure of the set of singular points at the first potential blow-up time is equal to zero. For stationary high dimensional Navier-Stokes equations, Struwe [17] proved that suitable weak solutions are regular outside a singular set of zero 1D Hausdorff measure in $\mathbb{R}^{5}$, and Kang [7] improved Struwe's result up to the boundary for a smooth domain $\Omega \subset \mathbb{R}^{5}$. Recently, the first author and Strain [3] studied the partial regularity for suitable weak solutions of $6 \mathrm{D}$ stationary Navier-Stokes equations, and proved that solutions are regular outside a singular set of zero 2D Hausdorff measure. Based on Campanato's approach, the main idea in [3] is to first establish a weak decay estimate of certain scale-invariant quantities, and then successively improve this decay estimate by using a bootstrap argument and the elliptic regularity theory.

Because time corresponds two space dimensions, in some sense the 4D nonstationary Navier-Stokes equations is similar to 6D stationary problem. Given the result in [3], it is natural to ask whether Caffarelli-Kohn-Nirenberg's theorem can be extended the $4 \mathrm{D}$ non-stationary case. Here the main difficulty stems from the fact that certain compactness arguments appeared, for instance, in the original paper $[\mathbf{1}]$ as well as $[\mathbf{1 2}, \mathbf{1 0}]$ break down in the $4 \mathrm{D}$ case. We note that the results obtained in [2] cannot be considered as a genuine extension of the theorem, as the 
set of singular points is only estimated at the first blow-up time for local smooth solutions. The objective of this paper is to give a complete answer to this question.

We state our main results, where we use some notation introduced at the beginning of the next section.

TheOrem 1.1. Let $\Omega$ be an open set in $\mathbb{R}^{4}$ and $f \in L_{6, l o c}\left(Q_{T}\right)$. Let $(u, p)$ be a pair of suitable weak solution of (1.1)-(1.2) in $Q_{T}$. There is a positive number $\varepsilon_{0}$ satisfying the following property. Assume that for a point $z_{0} \in Q_{T}$, the inequality

$$
\limsup _{r \searrow 0} E(r) \leq \varepsilon_{0}
$$

holds. Then $z_{0}$ is a regular point.

TheOREM 1.2. Let $\Omega$ be an open set in $\mathbb{R}^{4}$ and $f \in L_{6, l o c}\left(Q_{T}\right)$. Let $(u, p)$ be a pair of suitable weak solution of (1.1)-(1.2) in $Q_{T}$. There is a positive number $\varepsilon_{0}$ satisfying the following property. Assume that for a point $z_{0} \in Q_{T}$ and for some $\rho_{0}>0$ such that $Q\left(z_{0}, \rho_{0}\right) \subset Q_{T}$ and

$$
C\left(\rho_{0}\right)+D\left(\rho_{0}\right)+F\left(\rho_{0}\right)+G\left(\rho_{0}\right) \leq \varepsilon_{0} .
$$

Then $z_{0}$ is a regular point.

TheOREM 1.3. Let $\Omega$ be an open set in $\mathbb{R}^{4}$ and $f \in L_{6, l o c}\left(Q_{T}\right)$. Let $(u, p)$ be a pair of suitable weak solution of (1.1)-(1.2) in $Q_{T}$. Then the $2 D$ Hausdorff measure of the set of singular points in $Q_{T}$ is equal to zero.

In [2], local-in-time smooth solutions to $4 \mathrm{D}$ Navier-Stokes equations were considered, and the main idea is to apply Schoen's trick to get a priori estimates at the first potential blow-up time. In our paper, we shall consider suitable weak solutions, and thus Schoen's trick is no longer applicable. Our proofs exploit the aforementioned idea in [3] and use Campanato's approach. There are two main differences between our problem with the one in $[\mathbf{3}]$. The first one is that we do not have the same end-point Sobolev embedding inequality used in [3]. To this end, we introduce an additional scale-invariant quantity $F$, which is a mixed space-time norm of the pressure $p$, and use an interpolation inequality. As a consequence, we cannot achieve the same optimal decay rate as in $[\mathbf{3}]$. Nevertheless, it turns out that the decay rate, although not optimal, still suffices for our purpose in the subsequent step. The other difference is that, as our problem is time-dependent, we cannot use the elliptic regularity theory to improve the decay rate in the final step as in [3]. Naturally, we appeal to the parabolic regularity theory instead as well as a Poincaré type inequality for solutions to divergence form parabolic equations. We note that our definition of the scale-invariant quantity involving the pressure is different from those in $[\mathbf{2}]$ and $[\mathbf{3}]$. This makes the proof below slightly simpler.

It remains an interesting open problem whether a similar result can be obtained for five or higher dimensional non-stationary Navier-Stokes equations. It seems to us that four is the highest dimension to which our approach (or any existing approach) applies. In fact, by the embedding theorem, we have

$$
L_{\infty}\left((0, T) ; L_{2}(\Omega)\right) \cap L_{2}\left((0, T) ; W_{2}^{1}(\Omega)\right) \hookrightarrow L_{2(d+2) / d}((0, T) \times \Omega),
$$

which implies nonlinear term in the energy inequality cannot be controlled by the energy norm alone when $d \geq 5$.

We organize this paper as follows: In Section 2, we introduce some scaleinvariant quantities and the setting which will be used throughout the paper. In 
Section 3, we prove our results in three steps. In the first step, we give some estimates of the scale-invariant quantities, which essentially follow from the argument in [2]. In the second step, we establish a weak decay estimate of certain scaleinvariant quantities based on the estimate we proved in the first step by using an iteration method. In the last step, we improve the decay estimate by a bootstrap argument, and apply the parabolic regularity theory to get a good estimate of the $L_{3 / 2}$-mean oscillations of $u$, which yields the Hölder continuity of $u$ according to Campanato's characterization of Hölder continuous functions.

\section{Notation and Setting}

In this section, we will introduce the notation which will be used throughout the article. Let $\Omega$ be a domain in some finite-dimensional space. Denote $L_{p}\left(\Omega ; \mathbb{R}^{n}\right)$ and $W_{p}^{k}\left(\Omega ; \mathbb{R}^{n}\right)$ to be the usual Lebesgue and Sobolev spaces of functions from $\Omega$ into $\mathbb{R}^{n}$. Let $p \in(1, \infty)$ and $-\infty \leq S<T \leq \infty$. We denote $\mathcal{H}_{p}^{1}$ to be the solution spaces for divergence form parabolic equations. Precisely,

$$
\mathcal{H}_{p}^{1}(\Omega \times(S, T))=\left\{u: u, D u \in L_{p}(\Omega \times(S, T)), u_{t} \in \mathbb{H}_{p}^{-1}(\Omega \times(S, T)\},\right.
$$

where $\mathbb{H}_{p}^{-1}(\Omega \times(S, T))$ is the space consisting of all generalized functions $v$ satisfying

$$
\inf \left\{\|f\|_{L_{p}(\Omega \times(S, T))}+\|g\|_{L_{p}(\Omega \times(S, T))} \mid v=\nabla \cdot g+f\right\}<\infty \text {. }
$$

We shall use the following notation of spheres, balls, parabolic cylinders, and parabolic boundary

$$
\begin{aligned}
& S\left(x_{0}, r\right)=\left\{x \in \mathbb{R}^{4}|| x-x_{0} \mid=r\right\}, \quad S(r)=S(0, r), \quad S=S(1) ; \\
& B\left(x_{0}, r\right)=\left\{x \in \mathbb{R}^{4}|| x-x_{0} \mid<r\right\}, \quad B(r)=B(0, r), \quad B=B(1) ; \\
& Q\left(z_{0}, r\right)=B\left(x_{0}, r\right) \times\left(t_{0}-r^{2}, t_{0}\right), \quad Q(r)=Q(0, r), \quad Q=Q(1) ; \\
& \partial_{p} Q\left(z_{0}, r\right)=\left(S\left(x_{0}, r\right) \times\left[t_{0}-r^{2}, t_{0}\right)\right) \cup\left\{\left(t_{0}-r^{2}, y\right) \mid y \in B\left(x_{0}, r\right)\right\},
\end{aligned}
$$

where $z_{0}=\left(x_{0}, t_{0}\right)$.

We also denote mean values of summable functions as follows:

$$
\begin{aligned}
{[u]_{x_{0}, r}(t) } & =\frac{1}{|B(r)|} \int_{B\left(x_{0}, r\right)} u(x, t) d x \\
(u)_{z_{0}, r} & =\frac{1}{|Q(r)|} \int_{Q\left(z_{0}, r\right)} u d z
\end{aligned}
$$

Here $|A|$ as usual denotes the Lebesgue measure of the set $A$. 
Now we introduce the following quantities:

$$
\begin{aligned}
& A(r)=A\left(r, z_{0}\right)=\operatorname{esssup}_{t_{0}-r^{2} \leq t \leq t_{0}} \frac{1}{r^{2}} \int_{B\left(x_{0}, r\right)}|u(x, t)|^{2} d x, \\
& E(r)=E\left(r, z_{0}\right)=\frac{1}{r^{2}} \int_{Q\left(z_{0}, r\right)}|\nabla u|^{2} d z, \\
& C(r)=C\left(r, z_{0}\right)=\frac{1}{r^{3}} \int_{Q\left(z_{0}, r\right)}|u|^{3} d z, \\
& D(r)=D\left(r, z_{0}\right)=\frac{1}{r^{3}} \int_{Q\left(z_{0}, r\right)}\left|p-[p]_{x_{0}, r}\right|^{3 / 2} d z, \\
& F(r)=F\left(r, z_{0}\right)=\frac{1}{r^{2}}\left[\int_{t_{0}-r^{2}}^{t_{0}}\left(\int_{B\left(x_{0}, r\right)}\left|p-[p]_{x_{0}, r}\right|^{1+\alpha} d x\right)^{\frac{1}{2 \alpha}} d t\right]^{\frac{2 \alpha}{1+\alpha}}, \\
& G(r)=G\left(r, z_{0}\right)=r^{4}\left[\int_{Q\left(z_{0}, r\right)}|f|^{6} d z\right]^{\frac{1}{3}},
\end{aligned}
$$

where $\alpha \in(0,1)$ is a number to be specified later. Notice that all these quantities are invariant under the natural scaling:

$$
\begin{aligned}
& u_{\lambda}(x, t)=\lambda u\left(\lambda x, \lambda^{2} t\right), \\
& p_{\lambda}(x, t)=\lambda^{2} p\left(\lambda x, \lambda^{2} t\right), \\
& f_{\lambda}(x, t)=\lambda^{3} f\left(\lambda x, \lambda^{2} t\right) .
\end{aligned}
$$

We are going to estimate them in Section 3. We point out that the quantity $F$ is auxiliary and will only be used in the first two steps of the proof in order to give a weak decay estimate of other quantities.

We finish this short section by introducing a pressure decomposition which would play a important role in our proof. Let $\eta(x)$ be a smooth function on $\mathbb{R}^{4}$ supported in the unit ball $B(1), 0 \leq \eta \leq 1$ and $\eta \equiv 1$ on $\bar{B}(2 / 3)$. Let $z_{0}$ be a given point in $Q_{T}$ and $r>0$ a real number such that $Q\left(z_{0}, r\right) \subset Q_{T}$. It's known that for a.e. $t \in\left(t_{0}-r^{2}, t_{0}\right)$, in the sense of distribution, one has

$$
\begin{aligned}
\Delta p & =-\partial_{i} \partial_{j}\left(u_{i} u_{j}\right)+\nabla \cdot f \\
& =-\partial_{i} \partial_{j}\left(\left(u_{i}-\left[u_{i}\right]_{x_{0}, r / 2}\right)\left(u_{j}-\left[u_{j}\right]_{x_{0}, r / 2}\right)\right)+\nabla \cdot f \text { in } B\left(x_{0}, r\right) .
\end{aligned}
$$

For these $t$, we consider the decomposition

$$
p=\tilde{p}_{x_{0}, r}+h_{x_{0}, r} \text { in } B\left(x_{0}, r\right),
$$

where $\tilde{p}_{x_{0}, r}$ is the Newtonian potential of

$$
-\partial_{i} \partial_{j}\left(\left(u_{i}-\left[u_{i}\right]_{x_{0}, r / 2}\right)\left(u_{j}-\left[u_{j}\right]_{x_{0}, r / 2}\right) \eta\left(2\left(x-x_{0}\right) / r\right)\right)+\nabla \cdot\left(f \eta\left(2\left(x-x_{0}\right) / r\right)\right) .
$$

Then $h_{x_{0}, r}$ is harmonic in $B\left(x_{0}, r / 3\right)$.

\section{The proof}

In our proof of the main results, we will make use of the following well-known interpolation inequality. 
LEMMA 3.1. For any function $u \in W_{2}^{1}\left(\mathbb{R}^{4}\right)$ and real numbers $q \in[2,4]$ and $r>0$

$$
\begin{aligned}
\int_{B_{r}}|u|^{q} d x \leq N(q)\left[\left(\int_{B_{r}}|\nabla u|^{2} d x\right)^{q-2}\left(\int_{B_{r}}|u|^{2} d x\right)^{2-q / 2}\right. & \\
& \left.+r^{-2(q-2)}\left(\int_{B_{r}}|u|^{2} d x\right)^{q / 2}\right] .
\end{aligned}
$$

Let $\mathcal{L}:=\partial_{t}-\partial_{i}\left(a_{i j} \partial_{j}\right)$ be a (possibly degenerate) divergence form parabolic operator with measurable coefficients which are bounded by a constant $K>0$. We will use the following Poincaré type inequality for solutions to parabolic equations. See, for instance, [8, Lemma 3.1].

Lemma 3.2. Let $z_{0} \in \mathbb{R}^{d+1}, p \in(1, \infty), r \in(0, \infty), u \in \mathcal{H}_{p, l o c}^{1}\left(\mathbb{R}^{d+1}\right), g=$ $\left(g_{1}, \ldots, g_{d}\right), f \in L_{p, l o c}\left(\mathbb{R}^{d+1}\right)$. Suppose that $u$ is a weak solution to $\mathcal{L} u=\nabla \cdot g+f$ in $Q\left(z_{0}, r\right)$. Then we have

$$
\int_{Q\left(z_{0}, r\right)}\left|u(t, x)-(u)_{z_{0}, r}\right|^{p} d z \leq N r^{p} \int_{Q\left(z_{0}, r\right)}\left(|\nabla u|^{p}+|g|^{p}+r^{p}|f|^{p}\right) d z,
$$

where $N=N(d, K, p)$.

We shall prove the main theorems in three steps.

3.1. Step 1. First, we control the quantities $A, C, D, F$ in a smaller ball by their values in a larger ball under the assumption that $E$ is sufficiently small. Here we follow the arguments in $[\mathbf{2}]$, which in turn used some ideas in $[\mathbf{1 0}, \mathbf{1 2}]$.

Lemma 3.3. Suppose $\gamma \in(0,1), \rho>0$ are constants and $Q\left(z_{0}, \rho\right) \subset Q_{T}$. Then we have

$$
C(\gamma \rho) \leq N\left[\gamma^{-3} A^{1 / 2}(\rho) E(\rho)+\gamma^{-9 / 2} A^{3 / 4}(\rho) E^{3 / 4}(\rho)+\gamma C(\rho)\right],
$$

where $N$ is a constant independent of $\gamma, \rho$, and $z_{0}$.

The proof can be found in [2].

LEMMA 3.4. Suppose that $\alpha \in[1 / 11,1 / 2], \gamma \in(0,1 / 8], \rho>0$ are constants and $Q\left(z_{0}, \rho\right) \subset Q_{T}$. Then for any $z_{1} \in Q\left(z_{0}, \rho / 8\right)$ we have

$$
F\left(\gamma \rho, z_{1}\right) \leq N(\alpha)\left[\gamma^{-2} A^{\frac{1-\alpha}{1+\alpha}}\left(\frac{\rho}{2}\right) E^{\frac{2 \alpha}{1+\alpha}}\left(\frac{\rho}{2}\right)+\gamma^{\frac{3-\alpha}{1+\alpha}} F(\rho)+\gamma^{-2} G^{1 / 2}(\rho)\right]
$$

where $N(\alpha)$ is a constant independent of $\gamma, \rho$ and $z_{0}$. In particular, for $\alpha=1 / 2$ we have

$$
D\left(\gamma \rho, z_{1}\right) \leq N\left[\gamma^{-3} A^{1 / 2}\left(\frac{\rho}{2}\right) E\left(\frac{\rho}{2}\right)+\gamma^{5 / 2} D(\rho)+\gamma^{-3} G^{3 / 4}(\rho)\right]
$$

Moreover, it holds that

$$
\begin{aligned}
& D\left(\gamma \rho, z_{1}\right) \\
& \leq N(\alpha)\left[\gamma^{-3}\left(A\left(\frac{\rho}{2}\right)+E\left(\frac{\rho}{2}\right)\right)^{3 / 2}+\gamma^{\frac{9-3 \alpha}{2+2 \alpha}} F^{3 / 2}(\rho)+\gamma^{-3} G^{3 / 4}(\rho)\right] .
\end{aligned}
$$


Proof. First, we assume $1 / 3 \leq \alpha \leq 1 / 2$. Denote $r=\gamma \rho$. Recall the decomposition of $p$ introduced in (2.1) and the definition of $\eta$. By using the CalderónZygmund estimate, Lemma 3.1 with $q=2(1+\alpha)$, the Poincaré inequality, and the Sobolev embedding inequality, one has

$$
\begin{aligned}
& \int_{B\left(x_{0}, r\right)}\left|\tilde{p}_{x_{0}, r}\right|^{1+\alpha} d x \\
& \leq N \int_{B\left(x_{0}, r / 2\right)}\left|u-[u]_{x_{0}, r / 2}\right|^{2(1+\alpha)} d x \\
& \quad+N \int_{B\left(x_{0}, r\right)}\left|\Delta^{-1} \nabla \cdot\left(f \eta\left(2\left(x-x_{0}\right) / r\right)\right)\right|^{1+\alpha} d x \\
& \leq N\left(\int_{B\left(x_{0}, r / 2\right)}|\nabla u|^{2} d x\right)^{2 \alpha}\left(\int_{B\left(x_{0}, r / 2\right)}\left|u-[u]_{x_{0}, r / 2}\right|^{2} d x\right)^{1-\alpha} \\
& +N r^{-4 \alpha}\left(\int_{B\left(x_{0}, r / 2\right)}\left|u-[u]_{x_{0}, r / 2}\right|^{2} d x\right)^{1+\alpha}+N\left(\int_{B\left(x_{0}, r / 2\right)}|f|^{\frac{4+4 \alpha}{5+\alpha}} d x\right)^{\frac{5+\alpha}{4}} \\
& \leq N\left(\int_{B\left(x_{0}, r / 2\right)}|\nabla u|^{2} d x\right)^{2 \alpha}\left(\int_{B\left(x_{0}, r / 2\right)}|u|^{2} d x\right)^{1-\alpha} \\
& \quad+N\left(\int_{B\left(x_{0}, r / 2\right)}|f|^{\frac{4+4 \alpha}{5+\alpha}} d x\right)^{\frac{5+\alpha}{4}},
\end{aligned}
$$

where $\frac{4+4 \alpha}{5+\alpha} \geq 1$. Here we also used the obvious inequality

$$
\int_{B\left(x_{0}, r / 2\right)}\left|u-[u]_{x_{0}, r / 2}\right|^{2} d x \leq \int_{B\left(x_{0}, r / 2\right)}|u|^{2} d x .
$$

Similarly,

$$
\begin{aligned}
\int_{B\left(x_{0}, \rho\right)}\left|\tilde{p}_{x_{0}, \rho}\right|^{1+\alpha} d x \leq & N\left(\int_{B\left(x_{0}, \rho / 2\right)}|\nabla u|^{2} d x\right)^{2 \alpha}\left(\int_{B\left(x_{0}, \rho / 2\right)}|u|^{2} d x\right)^{1-\alpha} \\
& +N\left(\int_{B\left(x_{0}, \rho / 2\right)}|f|^{\frac{4+4 \alpha}{5+\alpha}} d x\right)^{\frac{5+\alpha}{4}} .
\end{aligned}
$$

Since $h_{x_{0}, \rho}$ is harmonic in $B\left(x_{0}, \rho / 3\right)$, any Sobolev norm of $h_{x_{0}, \rho}$ in a smaller ball can be estimated by its $L_{p}$ norm in $B\left(x_{0}, \rho / 3\right)$ for any $p \in[1, \infty]$. Thus, by using the Poincaré inequality one can obtain, for a.e. $t$,

$$
\begin{aligned}
& \int_{B\left(x_{1}, r\right)}\left|h_{x_{0}, \rho}-\left[h_{x_{0}, \rho}\right]_{x_{1}, r}\right|^{1+\alpha} d x \\
& \leq N r^{1+\alpha} \int_{B\left(x_{1}, r\right)}\left|\nabla h_{x_{0}, \rho}\right|^{1+\alpha} d x \\
& \leq N r^{5+\alpha} \sup _{B\left(x_{1}, r\right)}\left|\nabla h_{x_{0}, \rho}\right|^{1+\alpha} \\
& \leq N\left(\frac{r}{\rho}\right)^{5+\alpha} \int_{B\left(x_{0}, \rho / 3\right)}\left|h_{x_{0}, \rho}(x, t)-[p]_{x_{0}, \rho}\right|^{1+\alpha} d x \\
& \leq N\left(\frac{r}{\rho}\right)^{5+\alpha}\left[\int_{B\left(x_{0}, \rho\right)}\left|p(x, t)-[p]_{x_{0}, \rho}\right|^{1+\alpha}+\left|\tilde{p}_{x_{0}, \rho}(x, t)\right|^{1+\alpha} d x\right] .
\end{aligned}
$$


Since $\tilde{p}_{x_{0}, r}+h_{x_{0}, r}=p=\tilde{p}_{x_{0}, \rho}+h_{x_{0}, \rho}$ in $B\left(x_{1}, r\right)$, from (3.5), (3.6), and (3.7) we get, for a.e. $t$,

$$
\begin{aligned}
& \int_{B\left(x_{1}, r\right)}\left|p(x, t)-[p]_{x_{1}, r}\right|^{1+\alpha} d x \\
& \leq \int_{B\left(x_{1}, r\right)}\left|\tilde{p}_{x_{0}, \rho}-\left[\tilde{p}_{x_{0}, \rho}\right]_{x_{1}, r}\right|^{1+\alpha} d x+\int_{B\left(x_{1}, r\right)}\left|h_{x_{0}, \rho}-\left[h_{x_{0}, \rho}\right]_{x_{1}, r}\right|^{1+\alpha} d x \\
& \leq \int_{B\left(x_{1}, r\right)}\left|\tilde{p}_{x_{0}, \rho}\right|^{1+\alpha} d x+\int_{B\left(x_{1}, r\right)}\left|h_{x_{0}, \rho}-\left[h_{x_{0}, \rho}\right]_{x_{1}, r}\right|^{1+\alpha} d x \\
& \leq N\left(\int_{B\left(x_{0}, \rho / 2\right)}|\nabla u|^{2} d x\right)^{2 \alpha}\left(\int_{B\left(x_{0}, \rho / 2\right)}|u|^{2} d x\right)^{1-\alpha} \\
& +N\left(\frac{r}{\rho}\right)^{5+\alpha} \int_{B\left(x_{0}, \rho\right)}\left|p(x, t)-[p]_{x_{0}, \rho}\right|^{1+\alpha} d x+N\left(\int_{B\left(x_{0}, \rho\right)}|f|^{\frac{4+4 \alpha}{5+\alpha}} d x\right)^{\frac{5+\alpha}{4}} .
\end{aligned}
$$

Raising to power $\frac{1}{2 \alpha}$, integrating with respect to $t$ in $\left(t_{1}-r^{2}, t_{1}\right)$, and using Hölder's inequality complete the proof of (3.2) and (3.3).

In the case $1 / 11 \leq \alpha<1 / 3$, we cannot use the Sobolev embedding inequality directly in (3.5) because $\frac{4+4 \alpha}{5+\alpha}<1$. However, since $\eta$ has compact support, by using Hölder's inequality, we can get

$$
\begin{aligned}
& N \int_{B\left(x_{0}, r\right)}\left|\Delta^{-1} \nabla \cdot\left(f \eta\left(2\left(x-x_{0}\right) / r\right)\right)\right|^{1+\alpha} d x \\
& \leq N r^{4\left(1-\frac{1+\alpha}{1+\beta}\right)}\left(\int_{B\left(x_{0}, r\right)}\left|\Delta^{-1} \nabla \cdot\left(f \eta\left(2\left(x-x_{0}\right) / r\right)\right)\right|^{1+\beta} d x\right)^{\frac{1+\alpha}{1+\beta}} \\
& \leq N r^{4\left(1-\frac{1+\alpha}{1+\beta}\right)}\left(\int_{B\left(x_{0}, r / 2\right)}|f|^{\frac{4+4 \beta}{5+\beta}} d x\right)^{\frac{5+\beta}{4} \frac{1+\alpha}{1+\beta}}
\end{aligned}
$$

where $1 / 3 \leq \beta \leq 1 / 2$. Noting that $\frac{4+4 \beta}{5+\beta}<6$ and $\frac{1+\alpha}{2 \alpha} \leq 6$, we then prove (3.2) in the same way as the case $1 / 3 \leq \alpha \leq 1 / 2$.

To prove (3.4), we use a slightly different estimate from (3.7). Again, since $h$ is harmonic in $B\left(x_{0}, \rho / 3\right)$, we have

$$
\begin{aligned}
& \int_{B\left(x_{1}, r\right)}\left|h_{x_{0}, \rho}-\left[h_{x_{0}, \rho}\right]_{x_{1}, r}\right|^{3 / 2} d x \\
& \leq N r^{3 / 2} \int_{B\left(x_{1}, r\right)}\left|\nabla h_{x_{0}, \rho}\right|^{3 / 2} d x \\
& \leq N r^{11 / 2} \sup _{B\left(x_{1}, r\right)}\left|\nabla h_{x_{0}, \rho}\right|^{3 / 2} \\
& \leq N \frac{r^{11 / 2}}{\rho^{3 / 2+6 /(1+\alpha)}}\left[\int_{B\left(x_{0}, \rho / 3\right)}\left|h_{x_{0}, \rho}(x, t)-[p]_{x_{0}, \rho}\right|^{1+\alpha} d x\right]^{\frac{3}{2(1+\alpha)}} \\
& \leq N \frac{r^{11 / 2}}{\rho^{3 / 2+6 /(1+\alpha)}}\left\{\left[\int_{B\left(x_{0}, \rho\right)}\left|p(x, t)-[p]_{x_{0}, \rho}\right|^{1+\alpha} d x\right]^{\frac{3}{2(1+\alpha)}}\right. \\
& \left.+\left[\int_{B\left(x_{0}, \rho\right)}\left|\tilde{p}_{x_{0}, \rho}(x, t)\right|^{1+\alpha} d x\right]^{\frac{3}{2(1+\alpha)}}\right\} .
\end{aligned}
$$


Similar to (3.8), we obtain, for a.e. $t$,

$$
\begin{aligned}
& \int_{B\left(x_{1}, r\right)}\left|p(x, t)-[p]_{x_{1}, r}\right|^{3 / 2} d x \\
& \leq N\left(\int_{B\left(x_{0}, \rho / 2\right)}|\nabla u|^{2} d x\right)\left(\int_{B\left(x_{0}, \rho / 2\right)}|u|^{2} d x\right)^{1 / 2} \\
& \quad+N \frac{r^{11 / 2}}{\rho^{3 / 2+6 /(1+\alpha)}}\left\{\left[\int_{B\left(x_{0}, \rho\right)}\left|p(x, t)-[p]_{x_{0}, \rho}\right|^{1+\alpha} d x\right]^{\frac{3}{2(1+\alpha)}}\right. \\
& \left.+\left(\int_{B\left(x_{0}, \rho\right)}|\nabla u(x, t)|^{2} d x\right)^{\frac{3 \alpha}{1+\alpha}}\left(\int_{B\left(x_{0}, \rho\right)}|u(x, t)|^{2} d x\right)^{\frac{3(1-\alpha)}{2(1+\alpha)}}\right\} \\
& \quad+N\left(\int_{B\left(x_{0}, \rho\right)}|f|^{\frac{12}{11}} d x\right)^{\frac{11}{8}} .
\end{aligned}
$$

Integrating with respect to $t$ in $\left(t_{1}-r^{2}, t_{1}\right)$ and applying Hölder's inequality complete the proof of (3.4).

Lemma 3.5. Suppose that $\theta \in(0,1 / 2], \rho>0$ are constants and $Q\left(z_{0}, \rho\right) \subset Q_{T}$. Then we have

$$
A(\theta \rho)+E(\theta \rho) \leq N \theta^{-2}\left[C^{2 / 3}(\rho)+C(\rho)+C^{1 / 3}(\rho) D^{2 / 3}(\rho)+G(\rho)\right] .
$$

In particular, when $\theta=1 / 2$ we have

$$
A(\rho / 2)+E(\rho / 2) \leq N\left[C^{2 / 3}(\rho)+C(\rho)+C^{1 / 3}(\rho) D^{2 / 3}(\rho)+G(\rho)\right] .
$$

Proof. Let $r=\theta \rho$. In the energy inequality (1.3), we set $t=t_{0}$ and choose a suitable smooth cut-off function $\psi$ such that

$$
\begin{array}{r}
\psi \equiv 0 \text { in } Q_{t_{0}} \backslash Q\left(z_{0}, \rho\right), \quad 0 \leq \psi \leq 1 \text { in } Q_{T}, \\
\psi \equiv 1 \text { in } Q\left(z_{0}, r\right), \quad|\nabla \psi|<N \rho^{-1}, \quad\left|\partial_{t} \psi\right|+\left|\nabla^{2} \psi\right| \leq N \rho^{-2} \text { in } Q_{t_{0}} .
\end{array}
$$

By using (1.3) and because $u$ is divergence free, we get

$$
\begin{aligned}
A(r)+2 E(r) \leq \frac{N}{r^{2}}\left[\frac{1}{\rho^{2}} \int_{Q\left(z_{0}, \rho\right)}|u|^{2} d z+\frac{1}{\rho} \int_{Q\left(z_{0}, \rho\right)}\left(|u|^{2}+\right.\right. & \left.2\left|p-[p]_{x_{0}, \rho}\right|\right)|u| d z \\
& \left.+\int_{Q\left(z_{0}, \rho\right)}|f||u| d z\right] .
\end{aligned}
$$

Using Hölder's inequality and Young's inequality, one can obtain

$$
\begin{aligned}
\int_{Q\left(z_{0}, \rho\right)} & |u|^{2} d z \leq\left(\int_{Q\left(z_{0}, \rho\right)}|u|^{3} d z\right)^{2 / 3}\left(\int_{Q\left(z_{0}, \rho\right)} d z\right)^{1 / 3} \leq \rho^{4} C^{2 / 3}(\rho) \\
& \int_{Q\left(z_{0}, \rho\right)}\left|p-[p]_{x_{0}, \rho}\right||u| d z \\
& \leq\left(\int_{Q\left(z_{0}, \rho\right)}\left|p-[p]_{x_{0}, \rho}\right|^{3 / 2} d z\right)^{2 / 3}\left(\int_{Q\left(z_{0}, \rho\right)}|u|^{3} d z\right)^{1 / 3} \\
& \leq N \rho^{3} D^{2 / 3}(\rho) C^{1 / 3}(\rho)
\end{aligned}
$$


and

$$
\begin{aligned}
\int_{Q\left(z_{0}, \rho\right)}|f||u| d z & \leq \rho^{2} \int_{Q\left(z_{0}, \rho\right)}|f|^{2} d z+\frac{1}{\rho^{2}} \int_{Q\left(z_{0}, \rho\right)}|u|^{2} d z \\
& \leq \rho^{6}\left[\int_{Q\left(z_{0}, \rho\right)}|f|^{6} d z\right]^{1 / 3}+\frac{1}{\rho^{2}} \int_{Q\left(z_{0}, \rho\right)}|u|^{2} d z
\end{aligned}
$$

Then the conclusion follows immediately.

As a conclusion, we can obtain

Proposition 3.6. For any $\varepsilon_{0}>0$, there exists $\varepsilon_{1}>0$ small such that for any $z_{0} \in Q_{T}$ satisfying

$$
\limsup _{r \searrow 0} E(r) \leq \varepsilon_{1}
$$

we can find $\rho_{0}$ sufficiently small such that

$$
A\left(\rho_{0}\right)+E\left(\rho_{0}\right)+C\left(\rho_{0}\right)+D\left(\rho_{0}\right)+F\left(\rho_{0}\right) \leq \varepsilon_{0} .
$$

Proof. First, we prove (3.11) without the presence of $F$ on the left-hand side. For a given point

$$
z_{0}=\left(x_{0}, t_{0}\right) \in Q_{T}
$$

satisfying (3.10), choose $\rho_{1}>0$ such that $Q\left(z_{0}, \rho_{1}\right) \subset Q_{T}$. Then for any $\rho \in\left(0, \rho_{1}\right]$ and $\gamma \in(0,1 / 8)$, by using (3.9) and Young's inequality,

$$
A(\gamma \rho)+E(\gamma \rho) \leq N\left[C^{2 / 3}(2 \gamma \rho)+C(2 \gamma \rho)+D(2 \gamma \rho)+G(2 \gamma \rho)\right] .
$$

Then, combining with (3.1) and (3.3) and using Young's inequality again, we have

$$
\begin{aligned}
& A(\gamma \rho)+E(\gamma \rho)+C(\gamma \rho)+D(\gamma \rho) \\
& \leq N\left[\gamma^{2 / 3} C^{2 / 3}(\rho)+\gamma^{5 / 2} D(\rho)+\gamma C(\rho)+\gamma A(\rho)\right] \\
& \quad+N \gamma^{-100}\left(E(\rho)+E^{3}(\rho)+G(\rho)\right)+N \gamma^{2 / 3} \\
& \leq N \gamma^{2 / 3}[A(\rho)+E(\rho)+C(\rho)+D(\rho)]+N \gamma^{2 / 3} \\
& \quad+N \gamma^{-100}\left(E(\rho)+E^{3}(\rho)+G(\rho)\right) .
\end{aligned}
$$

Since $f \in L_{6, \text { loc }}\left(Q_{T}\right)$, we have

$$
G(\rho) \leq\|f\|_{L_{6}\left(Q\left(z_{0}, \rho_{1}\right)\right)}^{2} \rho^{4} .
$$

It is easy to see that for any $\varepsilon_{0}>0$, there are sufficiently small real numbers $\gamma \leq 1 /(2 N)^{3 / 2}$ and $\varepsilon_{1}$ such that if (3.10) holds then for all small $\rho$ we have

$$
N \gamma^{2 / 3}+N \gamma^{-100}\left(E(\rho)+E^{3}(\rho)+G(\rho)\right)<\varepsilon_{0} / 2 .
$$

By using (3.12), we can obtain

$$
A\left(\rho_{0}\right)+C\left(\rho_{0}\right)+D\left(\rho_{0}\right) \leq \varepsilon_{0}
$$

for some $\rho_{0}>0$ small enough. To include $F$ in the estimate, it suffices to use (3.2). 
3.2. Step 2. In the second step, first we will estimate the values of $A, E, C$, and $F$ in a smaller ball by the values of themselves in a larger ball.

Lemma 3.7. Suppose that $\rho>0, \theta \in(0,1 / 16]$ are constants and $Q\left(z_{1}, \rho\right) \subset Q_{T}$. Then we have

$$
\begin{aligned}
A(\theta \rho)+E(\theta \rho) \leq N \theta^{2} A(\rho)+N \theta^{-3}[A(\rho)+ & E(\rho)+F(\rho)]^{3 / 2} \\
& +N \theta^{-3} G^{3 / 4}(\rho)+N \theta^{-6} G(\rho),
\end{aligned}
$$

where $N$ is a constant independent of $\rho, \theta$, and $z_{1}$.

Proof. Let $r=\theta \rho$. Define the backward heat kernel as

$$
\Gamma(x, t)=\frac{1}{(4 \pi)^{2}\left(r^{2}+t_{1}-t\right)^{2}} e^{-\frac{\left|x-x_{1}\right|^{2}}{4\left(r^{2}+t_{1}-t\right)}} .
$$

In the energy inequality (1.3) we put $t=t_{1}$ and choose $\psi=\Gamma \phi:=\Gamma \phi_{1}(x) \phi_{2}(t)$, where $\phi_{1}$ and $\phi_{2}$ are suitable smooth cut-off functions satisfying

$$
\begin{aligned}
& \phi_{1} \equiv 0 \text { in } \mathbb{R}^{4} \backslash B\left(x_{1}, \rho\right), \quad 0 \leq \phi_{1} \leq 1 \text { in } \mathbb{R}^{4}, \quad \phi_{1} \equiv 1 \text { in } B\left(x_{1}, \rho / 2\right), \\
& \phi_{2} \equiv 0 \text { in }\left(-\infty, t_{1}-\rho^{2}\right) \cup\left(t_{1}+\rho^{2},+\infty\right), \quad 0 \leq \phi_{2} \leq 1 \text { in } \mathbb{R}, \\
& \phi_{2} \equiv 1 \text { in }\left(t_{1}-\rho^{2} / 4, t_{1}+\rho^{2} / 4\right), \quad\left|\partial_{t} \phi_{2}\right| \leq N \rho^{-2} \text { in } \mathbb{R},
\end{aligned}
$$

By using the equality

$$
\Delta \Gamma+\Gamma_{t}=0
$$

we have

$$
\begin{aligned}
& \int_{B\left(x_{1}, \rho\right)}|u(x, t)|^{2} \Gamma(t, x) \phi(x, t) d x+2 \int_{Q\left(z_{1}, \rho\right)}|\nabla u|^{2} \Gamma \phi d z \\
& \leq \int_{Q\left(z_{1}, \rho\right)}\left\{|u|^{2}\left(\Gamma \phi_{t}+\Gamma \Delta \phi+2 \nabla \phi \nabla \Gamma\right)+\left(|u|^{2}+2 p\right) u \cdot(\Gamma \nabla \phi+\phi \nabla \Gamma)\right\} d z \\
& \quad+\int_{Q\left(z_{1}, \rho\right)} 2|f||u||\Gamma \phi| d z .
\end{aligned}
$$

With straightforward computations, it is easy to see the following three properties:

(i) For some constant $c>0$, on $\bar{Q}\left(z_{1}, r\right)$ it holds that

$$
\Gamma \phi=\Gamma \geq c r^{-4} \text {. }
$$

(ii) For any $z \in Q\left(z_{1}, \rho\right)$, we have

$$
|\Gamma(z) \phi(z)| \leq N r^{-4}, \quad|\phi(z) \nabla \Gamma(z)|+|\nabla \phi(z) \Gamma(z)| \leq N r^{-5} .
$$

(iii) For any $z \in Q\left(z_{1}, \rho\right) \backslash Q\left(z_{1}, r\right)$, we have

$$
\left|\Gamma(z) \phi_{t}(z)\right|+|\Gamma(z) \Delta \phi(z)|+|\nabla \phi \nabla \Gamma| \leq N \rho^{-6} .
$$

These properties together with (3.15) and (3.16) yield

$$
A(r)+E(r) \leq N\left[\theta^{2} A(\rho)+\theta^{-3}(C(\rho)+D(\rho))+\theta^{-6} G(\rho)\right] .
$$

Owing to (3.1) with $q=3$, we can get

$$
C(\rho / 8) \leq N C(\rho) \leq N[A(\rho)+E(\rho)]^{3 / 2} .
$$


By using (3.4) with $\gamma=1 / 8$, we have

$$
D(\rho / 8) \leq N[A(\rho)+E(\rho)+F(\rho)]^{3 / 2}+N G^{3 / 4}(\rho) .
$$

Upon combining (3.17) (with $\rho / 8$ in place of $\rho$ ) to (3.19) together, the lemma is proved.

Lemma 3.8. Suppose that $\rho>0$ is constant and $Q\left(z_{1}, \rho\right) \subset Q_{T}$. Then we can find $\theta_{1} \in(0,1 / 256]$ small such that

$$
\begin{aligned}
A\left(\theta_{1} \rho\right)+E\left(\theta_{1} \rho\right)+F\left(\theta_{1} \rho\right) & \leq \frac{1}{2}[A(\rho)+E(\rho)+F(\rho)] \\
& +N\left(\theta_{1}\right)[A(\rho)+E(\rho)+F(\rho)]^{3 / 2}+N\left(\theta_{1}\right)\left[G(\rho)+G^{1 / 2}(\rho)\right]
\end{aligned}
$$

where $N$ is a constant independent of $\rho$ and $z_{1}$.

Proof. Due to (3.2) and (3.14), for any $\gamma, \theta \in(0,1 / 16]$, we have

$$
\begin{aligned}
F(\gamma \theta \rho) \leq & N\left[\gamma^{-2}(A(\theta \rho)+E(\theta \rho))+\gamma^{(3-\alpha) /(1+\alpha)} F(\theta \rho)+\gamma^{-2} G^{1 / 2}(\theta \rho)\right] \\
\leq & N \gamma^{-2} \theta^{2} A(\rho)+\gamma^{(3-\alpha) /(1+\alpha)} \theta^{-2} F(\rho)+\gamma^{-2} G^{1 / 2}(\rho) \\
& +N \gamma^{-2} \theta^{-3}[A(\rho)+E(\rho)+F(\rho)]^{3 / 2}
\end{aligned}
$$

and

$$
\begin{aligned}
A(\gamma \theta \rho)+E(\gamma \theta \rho) \leq & N(\gamma \theta)^{2} A(\rho)+N(\gamma \theta)^{-3}[A(\rho)+E(\rho)+F(\rho)]^{3 / 2} \\
& +N(\gamma \theta)^{-6} G(\rho)+N(\gamma \theta)^{-3} G^{3 / 4}(\rho) .
\end{aligned}
$$

Now we set $\alpha=1 / 5$ such that $(3-\alpha) /(1+\alpha)=7 / 3>2$, and choose and fix $\gamma$ and $\theta$ sufficiently small such that

$$
N\left[\gamma^{-2} \theta^{2}+\gamma^{7 / 3} \theta^{-2}+\gamma^{2} \theta^{2}\right] \leq \frac{1}{2}
$$

Upon adding (3.21) and (3.22), we obtain

$$
\begin{aligned}
A(\gamma \theta \rho)+E(\gamma \theta \rho)+F(\gamma \theta \rho) & \leq \frac{1}{2} A(\rho)+N[A(\rho)+E(\rho)+F(\rho)]^{3 / 2} \\
& +N\left[G(\rho)+G^{1 / 2}(\rho)+G^{3 / 4}(\rho)\right],
\end{aligned}
$$

where $N$ only depends on $\theta$ and $\gamma$. After putting $\theta_{1}=\gamma \theta$, the lemma is proved.

In the next proposition we will study the decay property of $A, C, E$, and $F$ as the radius $\rho$ goes to zero.

Proposition 3.9. There exists $\varepsilon_{0}>0$ satisfying the following property. Suppose that for some $z_{0} \in Q_{T}$ and $\rho_{0}>0$ satisfying $Q\left(z_{0}, \rho_{0}\right) \subset Q_{T}$ we have

$$
C\left(\rho_{0}\right)+D\left(\rho_{0}\right)+F\left(\rho_{0}\right)+G\left(\rho_{0}\right) \leq \varepsilon_{0} .
$$

Then we can find $N>0$ and $\alpha_{0} \in(0,1)$ such that for any $\rho \in\left(0, \rho_{0} / 8\right)$ and $z_{1} \in Q\left(z_{0}, \rho / 8\right)$, the following inequality will hold uniformly

$$
A\left(\rho, z_{1}\right)+C\left(\rho, z_{1}\right)+E\left(\rho, z_{1}\right)+F\left(\rho, z_{1}\right)+D\left(\rho, z_{1}\right) \leq N \rho^{\alpha_{0}},
$$

where $N$ is a positive constant independent of $\rho$ and $z_{1}$. 
Proof. Fix the constant $\theta_{1} \in(0,1 / 256]$ from Lemma 3.8 and let $N\left(\theta_{1}\right)>0$ be the same constant from (3.20). Due to (3.9), (3.23), and (3.2), we first choose $\varepsilon^{\prime}>0$ and then $\varepsilon_{0}=\varepsilon_{0}\left(\varepsilon^{\prime}\right)>0$ sufficiently small such that,

$$
\begin{gathered}
N\left(\theta_{1}\right) \sqrt{\varepsilon^{\prime}} \leq 1 / 4, \quad N\left(\theta_{1}\right)\left(\varepsilon_{0}+\varepsilon_{0}^{1 / 2}\right) \leq \varepsilon^{\prime} / 2, \\
A\left(\rho_{0} / 2\right)+E\left(\rho_{0} / 2\right) \leq \frac{\varepsilon^{\prime}}{32},
\end{gathered}
$$

and for any $z_{1} \in Q\left(z_{0}, \rho_{0} / 8\right)$,

$$
F\left(\rho_{0} / 8, z_{1}\right) \leq N\left[A^{2 / 3}\left(\frac{\rho_{0}}{2}\right) E^{1 / 3}\left(\frac{\rho_{0}}{2}\right)+F\left(\rho_{0}\right)+G^{1 / 2}\left(\rho_{0}\right)\right] \leq \frac{\varepsilon^{\prime}}{32} .
$$

By using

$$
Q\left(z_{1}, \rho_{0} / 8\right) \subset Q\left(z_{0}, \rho_{0} / 2\right) \subset Q_{T},
$$

we then have

$$
\phi\left(\rho_{0}\right):=A\left(\rho_{0} / 8, z_{1}\right)+E\left(\rho_{0} / 8, z_{1}\right)+F\left(\rho_{0} / 8, z_{1}\right) \leq \varepsilon^{\prime} .
$$

From (3.20) and (3.25) with $\rho=\rho_{0} / 8$, we obtain inductively that

$$
\phi\left(\theta_{1}^{k} \rho_{0}\right)=A\left(\theta_{1}^{k} \rho_{0} / 8, z_{1}\right)+E\left(\theta_{1}^{k} \rho_{0} / 8, z_{1}\right)+F\left(\theta_{1}^{k} \rho_{0} / 8, z_{1}\right) \leq \varepsilon^{\prime}
$$

(holding for $k=1,2, \ldots)$. It then similarly follows from $(3.20)$ and $(3.25)$ that

$$
\phi\left(\theta_{1}^{k} \rho_{0}\right) \leq \frac{3}{4} \phi\left(\theta_{1}^{k-1} \rho_{0}\right)+N_{1}\left(\theta_{1}^{k-1} \rho_{0}\right)^{2},
$$

where we have used the estimate

$$
G\left(\theta_{1}^{k-1} \rho_{0} / 8, z_{1}\right)+G^{1 / 2}\left(\theta_{1}^{k-1} \rho_{0} / 8, z_{1}\right) \leq N\left(\|f\|_{L_{6}\left(Q\left(z_{0}, \rho_{0} / 2\right)\right.}\right)\left(\theta_{1}^{k-1} \rho_{0}\right)^{2} .
$$

By a standard iteration argument, we obtain the decay rate of $\phi$ as follows. We iterate (3.26) to obtain

$$
\phi\left(\theta_{1}^{k} \rho_{0}\right) \leq\left(\frac{3}{4}\right)^{k}\left[\phi\left(\rho_{0}\right)+\frac{2 N_{1}}{1-\theta_{1}} \rho_{0}^{2}\right],
$$

where we have used that $\theta_{1}<3 / 4$. Since $\rho \in\left(0, \rho_{0} / 8\right)$ we can find $k$ such that $\theta_{1}^{k} \frac{\rho_{0}}{8}<\rho \leq \theta_{1}^{k-1} \frac{\rho_{0}}{8}$. Then

$$
\begin{aligned}
A\left(\rho, z_{1}\right)+E\left(\rho, z_{1}\right)+F\left(\rho, z_{1}\right) & \leq N\left(\theta_{1}\right)\left(\frac{3}{4}\right)^{k}\left[\phi\left(\rho_{0}\right)+\frac{2 N_{1}}{1-\theta_{1}} \rho_{0}^{2}\right] \\
& \leq N \rho^{\alpha_{0}},
\end{aligned}
$$

where $N=N\left(\theta_{1}, \phi\left(\rho_{0}\right), N_{1}, \rho_{0}\right)$ and $\alpha_{0}=\frac{\log (3 / 4)}{\log \left(\theta_{1}\right)}>0$. This yields (3.24) for the terms $A, E$, and $F$. The inequality for $C\left(\rho, z_{1}\right)$ follows from (3.18) and the inequality for $D\left(\rho, z_{1}\right)$ follows by (3.4). 
3.3. Step 3. In the final step, we are going to use a bootstrap argument to successively improve the decay estimate (3.24). However, as we will show below, the bootstrap argument itself only gives the decay of $E(\rho)$ no more than $\rho^{5 / 3}$, for instance, one can get an estimate like

$$
\int_{Q\left(z_{1}, \rho\right)}|\nabla u|^{2} d z \leq N \rho^{2+\frac{5}{3}}
$$

for any $\rho$ sufficiently small. Unfortunately, this decay estimate is not enough for the Hölder regularity of $u$ since the spatial dimension is four (so that we need the decay exponent $4+\varepsilon$ according to Morrey's lemma). We shall use the parabolic regularity theory to fill in this gap.

First we prove Theorem 1.2. We begin with the bootstrap argument. We shall choose an increasing sequence of real numbers $\left\{\alpha_{k}\right\}_{k=0}^{m} \in\left(\alpha_{0}, 5 / 3\right]$. Under the condition (3.24), we claim that the following estimates hold uniformly for all $\rho>0$ sufficiently small and $z_{1} \in Q\left(z_{0}, \rho_{0} / 8\right)$ over the range of $\left\{\alpha_{k}\right\}_{k=0}^{m}$ :

$$
A\left(\rho, z_{1}\right)+E\left(\rho, z_{1}\right) \leq N \rho^{\alpha_{k}}, C\left(\rho, z_{1}\right)+D\left(\rho, z_{1}\right) \leq N \rho^{3 \alpha_{k} / 2} .
$$

We prove this via iteration. The $k=0$ case for (3.27) was proved in (3.24) with a possibly different exponent $\alpha_{0}$.

Now we suppose (3.24) holds with the exponent $\alpha_{k}$. We first estimate $A\left(\rho, z_{1}\right)$ and $E\left(\rho, z_{1}\right)$. Let $\rho=\tilde{\theta} \tilde{\rho}$, where $\tilde{\theta}=\rho^{\mu}, \tilde{\rho}=\rho^{1-\mu}$ and $\mu \in(0,1)$ to be determined. We use (3.17), (3.27) (for $\alpha_{k}$ ), and (3.13) to obtain

$$
A(\rho)+E(\rho) \leq N \rho^{2 \mu} \rho^{\alpha_{k}(1-\mu)}+N \rho^{-3 \mu} \rho^{\frac{3}{2} \alpha_{k}(1-\mu)}+N \rho^{4(1-\mu)} \rho^{-6 \mu} .
$$

Setting $\mu=\frac{\alpha_{k}}{10+\alpha_{k}}$ gives (3.27) for $A(\rho)+E(\rho)$ with the exponent of

$$
\begin{aligned}
\tilde{\alpha}_{k+1} & :=\min \left\{2 \mu+\alpha_{k}(1-\mu), \frac{3}{2} \alpha_{k}(1-\mu)-3 \mu, 4(1-\mu)-6 \mu\right\} \\
& =\frac{12}{10+\alpha_{k}} \alpha_{k} \in\left(\alpha_{k}, 2\right) .
\end{aligned}
$$

The estimate in (3.27) (with $\left.\tilde{\alpha}_{k+1}\right)$ for $C\left(\rho, z_{1}\right)$ follows from (3.18). To prove the estimate in (3.27) (with $\alpha_{k+1}$ ) for $D\left(\rho, z_{1}\right)$ we use Lemma 3.4. From (3.3) and (3.13), we have

$$
D\left(\gamma \rho, z_{1}\right) \leq N\left[\gamma^{-3} \rho^{\frac{3}{2} \tilde{\alpha}_{k+1}}+\gamma^{5 / 2} D\left(\rho, z_{1}\right)+\gamma^{-3} \rho^{3}\right] .
$$

For any $r$ small, we take the supremum on both sides with respect to $\rho \in(0, r)$ and get

$$
\sup _{\rho \in(0, r]} D\left(\gamma \rho, z_{1}\right) \leq N \gamma^{-3} r^{\frac{3}{2}} \tilde{\alpha}_{k+1}+N \gamma^{5 / 2} \sup _{\rho \in(0, r]} D\left(\rho, z_{1}\right)+\gamma^{-3} r^{3}
$$

Set

$$
\alpha_{k+1}=\min \left\{\frac{5}{3}, \tilde{\alpha}_{k+1}\right\} .
$$

By using a well-known iteration argument, similar to (3.26), we obtain the estimate in (3.27) (with $\alpha_{k+1}$ ) for $D(\rho)$. Since $\alpha_{k+1} \leq \tilde{\alpha}_{k+1}$, the estimates in (3.27) for $A(\rho)+E(\rho)$ and $C(\rho)$ still holds with $\alpha_{k+1}$ in place of $\tilde{\alpha}_{k+1}$. Therefore, we have shown how to build the increasing sequence of $\left\{\alpha_{k}\right\}$ for which (3.27) holds. 
Note that

$$
2-\tilde{\alpha}_{k+1}=\frac{10}{10+\tilde{\alpha}_{k}}\left(2-\tilde{\alpha}_{k}\right) \leq \frac{10}{10+\alpha_{0}}\left(2-\tilde{\alpha}_{k}\right) .
$$

Thus, we can find a $m$ that $\alpha_{m}=\frac{5}{3}$ according to (3.28) because otherwise $\alpha_{k}=$ $\tilde{\alpha}_{k} \rightarrow 2$ as $k \rightarrow \infty$.

We have derived the following estimates via the bootstrap argument:

$$
\begin{aligned}
& \sup _{t_{1}-\rho^{2} \leq t \leq t_{1}} \int_{B\left(x_{1}, \rho\right)}|u(x, t)|^{2} d x \leq N \rho^{2+\frac{5}{3}}, \\
& \int_{Q\left(z_{1}, \rho\right)}|u|^{3}+\left|p-[p]_{x_{1}, \rho}\right|^{3 / 2} d z \leq N \rho^{3+\frac{5}{2}} .
\end{aligned}
$$

Next we rewrite (1.1) (in the weak sense) into

$$
\partial_{t} u_{i}-\Delta u_{i}=-\partial_{j}\left(u_{i} u_{j}\right)-\partial_{i} p+f_{i} .
$$

Finally, we use the parabolic regularity theory to improve the decay estimate of mean oscillations of $u$ and then complete the proof. Due to (3.29) and (3.30), there exists $\rho_{1} \in(\rho / 2, \rho)$ such that

$$
\int_{B\left(x_{1}, \rho_{1}\right)}\left|u\left(x, t_{1}-\rho_{1}^{2}\right)\right|^{2} d x \leq N \rho^{2+\frac{5}{3}}, \quad \int_{t_{1}-\rho_{1}^{2}}^{t_{1}} \int_{S\left(x_{1}, \rho_{1}\right)}|u|^{3} d x d t \leq N \rho^{2+\frac{5}{2}} .
$$

Let $v$ be the unique weak solution to the heat equation

$$
\partial_{t} v-\Delta v=0 \text { in } Q\left(z_{1}, \rho_{1}\right)
$$

with the boundary condition $v_{i}=u_{i}$ on $\partial_{p} Q\left(z_{1}, \rho_{1}\right)$. It follows from the standard estimates for the heat equation, Hölder's inequality, and (3.32) that

$$
\begin{aligned}
& \sup _{Q\left(z_{1}, \rho_{1} / 2\right)}|\nabla v| \\
& \leq N \rho_{1}^{-6} \int_{t_{1}-\rho_{1}^{2}}^{t_{1}} \int_{S\left(x_{1}, \rho_{1}\right)}|v| d x d t+N \rho_{1}^{-5} \int_{B\left(x_{1}, \rho_{1}\right)}\left|v\left(x, t_{1}-\rho_{1}^{2}\right)\right| d x \\
& \leq N \rho^{-2+\frac{5}{6}} .
\end{aligned}
$$

Denote $w=u-v$. Then $w$ satisfies the linear parabolic equation

$$
\partial_{t} w_{i}-\Delta w_{i}=-\partial_{j}\left(u_{i} u_{j}\right)-\partial_{i}\left(p-[p]_{x_{1}, \rho}\right)+f_{i}
$$

with the zero boundary condition. By the classical $L_{p}$ estimate for parabolic equations, we have

$$
\begin{aligned}
\|\nabla w\|_{L_{3 / 2}\left(Q\left(z_{1}, \rho_{1}\right)\right)} \leq N\left\||u|^{2}\right\|_{L_{3 / 2}\left(Q\left(z_{1}, \rho_{1}\right)\right)}+N\left\|p-[p]_{x_{1}, \rho}\right\|_{L_{3 / 2}\left(Q\left(z_{1}, \rho_{1}\right)\right)} \\
+N \rho_{1}\|f\|_{L_{3 / 2}\left(Q\left(z_{1}, \rho_{1}\right)\right)},
\end{aligned}
$$

which together with (3.30) and the condition $f \in L_{6, \text { loc }}$ yields

$$
\int_{Q\left(z_{1}, \rho_{1}\right)}|\nabla w|^{3 / 2} d z \leq N \rho^{11 / 2}
$$

Since $|\nabla u| \leq|\nabla w|+|\nabla v|$, we combine (3.33) and (3.34) to obtain, for any $r \in$ $(0, \rho / 4)$, that

$$
\int_{Q\left(z_{1}, r\right)}|\nabla u|^{3 / 2} d z \leq N \rho^{11 / 2}+r^{6} \rho^{-3+\frac{5}{4}} .
$$


Upon taking $r=\rho^{29 / 24} / 4$ (with $\rho$ small), we deduce

$$
\int_{Q\left(z_{1}, r\right)}|\nabla u|^{3 / 2} d z \leq N r^{\beta},
$$

where

$$
\beta=\frac{132}{29}>6-\frac{3}{2} \text {. }
$$

Since $u \in \mathcal{H}_{3 / 2 \text {,loc }}^{1}$ is a weak solution to (3.31), it then follows from Lemma 3.2, (3.35), and (3.30) with $r$ in place of $\rho$ that

$$
\begin{aligned}
& \int_{Q\left(z_{1}, r\right)}\left|u-(u)_{z_{1}, r}\right|^{3 / 2} d z \\
& \left.\leq N r^{3 / 2} \int_{Q\left(z_{1}, r\right)}|\nabla u|^{3 / 2}+\left(|u|^{2}\right)^{3 / 2}+\left|p-[p]_{x_{1}, r}\right|^{3 / 2}+r^{3 / 2}|f|^{3 / 2}\right) d z \\
& \leq N r^{\beta+3 / 2} .
\end{aligned}
$$

By Campanato's characterization of Hölder continuous functions (see, for instance, [11, Lemma 4.3]), $u$ is Hölder continuous in a neighborhood of $z_{0}$. This completes the proof of Theorem 1.2.

Theorem 1.1 then follows from Theorem 1.2 by applying Proposition 3.6. Finally, Theorem 1.3 is deduced from Theorem 1.1 by using a standard argument in the geometric measure theory, which is explained, for example, in [1].

Final Remarks: Finally we mention that in [6] Han and He studied the partial regularity for $4 \mathrm{D}$ MHD equations, which reduce to the Navier-Stokes equations by setting the magnetic field to be zero. However, it seems to us that their argument, in particular the proof of Lemma 2.6 there, does not seem to be complete.

After we finished this paper, we learned that a result similar to Theorem 1.1 was obtained recently in Wang and $\mathrm{Wu}[\mathbf{2 0}]$ by following the proof in [19]. Compared to $[6,20]$, apart from a standard argument to get some estimates of the scale-invariant quantities, our methodology is completely different. Our proof can also be adapted to other situations, for instance, the boundary partial regularity problems, which we plan to study in a subsequent paper [4].

\section{Acknowledgements.}

The authors are grateful to the referee for his helpful comments. H. Dong was partially supported by the NSF under agreement DMS-1056737. X. Gu was sponsored by the China Scholarship Council for one year study at Brown University and was partially supported by the NSFC (grant No. 11171072) and the Innovation Program of Shanghai Municipal Education Commission (grant No. 12ZZ012).

\section{References}

[1] L. Caffarelli, R. Kohn, L. Nirenberg, Partial regularity of suitable weak solutions of the Navier-stokes equations, Comm. Pure Appl. Math. 35 (1982), no. 6, 771-831.

[2] H. Dong, D. Du, Partial regularity of solutions to the four-dimensional Navier-Stokes equations at the first blow-up time, Comm. Math. Phys. 273 (2007), no. 3, 785-801.

[3] H. Dong, R. Strain, On partial regularity of steady-state solutions the 6D Navier-Stokes Equations, Indiana Univ. Math. J. 61 (2012), no. 6, 2211-2229.

[4] H. Dong, X. Gu, Boundary partial regularity for the high dimensional Navier-Stokes equations, preprint, arXiv:1309.3348. 
[5] S. Gustafson, K. Kang, T. Tsai, Interior regularity criteria for suitable weak solutions of the Navier-Stokes equations, Comm. Math. Phys. 273 (2007), no. 1, 161-176.

[6] P. Han, C. He, Partial regularity of suitable weak solutions to the four-dimensional incompressible magneto-hydrodynamic equations, Mathematical Methods in the Applied Sciences. 35 (2012), no. 11, 1335-1355.

[7] K. Kang, On regularity of stationary Stokes and Navier-Stokes equations near boundary, J. Math. Fluid Mech. 6 (2004), no. 1, 78-101.

[8] N. V. Krylov, Parabolic and elliptic equations with VMO coefficients, Comm. Partial Differential Equations 32 (2007), no. 1-3, 453-475.

[9] I. Kukavica, On partial regularity for the Navier-Stokes equations, Discrete Contin. Dyn. Syst. 21 (2008), no. 3, 717-728.

[10] O. Ladyzhenskaya, G. A. Seregin, On the partial regularity of suitable weak solutions to the three-dimensional Navier-Stokes equations, J. Math. Fluid Mech. 1 (1999), no. 4, 356-387.

[11] G. M. Lieberman, Second order parabolic differential equations, World Scientifc Publishing Co., Inc., River Edge, NJ, 1996.

[12] F. Lin, A new proof of the Caffarelli-Kohn-Nirenberg theorem, Comm. Pure. Appl. Math. 51 (1998), no. 3, 241-257.

[13] V. Scheffer, Partial regularity of solutions to the Navier-Stokes equations, Pacific J. Math. 66 (1976), no. 2, 532-522.

[14] V. Scheffer, Hausdorff measure and the Navier-Stokes equations, Comm. Math. Phys. 55 (1977), no. 2, 97-112.

[15] V. Scheffer, The Navier-Stokes equations in space dimension four, Comm. Math. Phys. 61 (1978), no. 1, 41-68.

[16] G. Seregin, Estimates of suitable weak solutions to the Navier-Stokes equations in critical Morrey spaces, Zapiski Nauchn. Seminar POMI 336 (2006), no. 2, 199-210.

[17] M. Struwe, On partial regularity results for the Navier-Stokes equations, Comm. Pure Appl. Math. 41 (1988), no. 4, 437-458.

[18] G. Tian, Z. Xin, Gradient estimation on Navier-Stokes equations, Comm. Anal. Geom. 7 (1999), no. 2, 221-257.

[19] A. Vasseur, A new proof of partial regularity of solutions to Navier-Stokes equations, NoDEA Nonlinear Differential Equations Appl. 14 (2007), no. 5-6, 753-785.

[20] Y. Wang, G. Wu, A unified proof on the partial regularity for suitable weak solutions of non-stationary and stationary Navier-Stokes equations, J. Differential Equations to appear, (2013).

Division of Applied Mathematics, Brown University, 182 George Street, ProviDENCE, RI 02912, USA

E-mail address: Hongjie_Dong@brown.edu

School of Mathematical Sciences, Fudan University, Shanghai 200433, People's RePUBLIC OF China

E-mail address: xumin_gu@brown.edu;11110180030@fudan.edu.cn 